\title{
Optically probing the fine structure of a single Mn atom in an InAs quantum dot
}

\author{
A. Kudelski, ${ }^{1}$ A. Lemaître,,${ }^{1,}$ A. Miard,${ }^{1}$ P. Voisin, ${ }^{1}$ T. C. M. Graham, ${ }^{2}$ R. J. Warburton, ${ }^{2}$ and O. Krebs ${ }^{1},{ }^{\dagger}$ \\ ${ }^{1}$ Laboratoire de Photonique et Nanostructures-CNRS, Route de Nozay, 91460 Marcoussis, France \\ ${ }^{2}$ School of Engineering and Physical Sciences, Heriot-Watt University, Edinburgh EH14 4AS, United Kingdom
}

(Dated: October 26, 2018)

\begin{abstract}
We report on the optical spectroscopy of a single InAs/GaAs quantum dot (QD) doped with a single $\mathrm{Mn}$ atom in a longitudinal magnetic field of a few Tesla. Our findings show that the Mn impurity is a neutral acceptor state $A^{0}$ whose effective spin $J=1$ is significantly perturbed by the QD potential and its associated strain field. The spin interaction with photo-carriers injected in the quantum dot is shown to be ferromagnetic for holes, with an effective coupling constant of a few hundreds of $\mu \mathrm{eV}$, but vanishingly small for electrons.

PACS numbers: 71.35.Pq, 78.67.Hc,75.75.+a,78.55.Cr
\end{abstract}

The spin state of a single magnetic impurity could be envisaged as a primary building block of a nanoscopic spin-based device 1, 22 in particular for the realization of quantum bits [3. However probing and manipulating such a system require extremely high sensitivity. Several techniques have been successfully developed over the last few years to address a single or few coupled spins: electrical detection [4, 5], scanning tunneling microscopy (STM) 6, 7, 8, 9, magnetic resonance force microscopic [10, optical spectroscopy 11]. Recently, Besombes et al. [12, 13, 14, 15] have investigated the spin state of a single $\mathrm{Mn}^{+2}$ ion embedded in a single II-VI self-assembled quantum dot (QD). In this system the magnetic impurity is an isoelectronic center in a $3 d^{5}$ configuration with spin $S=5 / 2$. The large exchange interaction between the spin of the photocreated carriers confined inside the dot and the Mn magnetic moment induces strong modifications of the QD photoluminescence (PL) spectrum: $2 S+1=6$ discrete lines are observed, reflecting the $\mathrm{Mn}$ spin state at the instant when the exciton recombines.

The case of the Mn ion is different in GaAs, since the impurity is an acceptor in this matrix with a rather large activation energy $(113 \mathrm{meV})$. Two types of $\mathrm{Mn}$ centers exist in GaAs, the $A^{0}$ and the $A^{-}$states. In low doped GaAs (below $10^{19} \mathrm{~cm}^{-2}$ ), the former is dominant. It corresponds to the $3 d^{5}+h$ configuration, where $h$ is a hole bound to the Mn ion with a Bohr radius around $1 \mathrm{~nm}$ [16. When considering a single $\mathrm{Mn}$ impurity in InAs QD several issues arise: the impurity configuration, its possible change when photo-carriers are captured, the influence on the binding energy of excitonic complexes, the strength and sign of the effective exchange interaction with each of the carriers (electron or hole) in the QD $S$-shell. In this Letter, we report the first evidences of a single $\mathrm{Mn}$ impurity in an individual InAs QD which enable us to answer most of the above questions. In particular, we find that the formation of excitons, biexciton and trions is weakly perturbed by the impurity center, whereas the effective exchange coupling with the Mn impurity (found in the $A^{0}$ configuration) is ferromagnetic for holes (a few $100 \mu \mathrm{eV}$ 's) and almost zero for the electrons.

The sample was grown by molecular beam epitaxy on a semi-insulating GaAs [001] substrate. The Mn-doped quantum layer was embedded inbetween an electron reservoir and a Schottky gate. This design gave us the possibility to observe both neutral and charged excitons. It consists of a $200 \mathrm{~nm}$ thick $n$-doped GaAs layer $\left(n=2 \times 10^{18} \mathrm{~cm}^{-2}\right)$ followed by a non-intentionally doped (n-i-d) $20 \mathrm{~nm}$ GaAs layer, the Mn-doped QD layer, and capped with a n-i-d GaAs $(30 \mathrm{~nm}) / \mathrm{Ga}_{0.7} \mathrm{Al}_{0.3} \mathrm{As}$ $(100 \mathrm{~nm}) /$ GaAs $(20 \mathrm{~nm})$ structure. The QD layer was formed by the deposition of 1.7 ML of InAs during $5 \mathrm{~s}$. The substrate temperature was set to $500{ }^{\circ} \mathrm{C}$ (optimal for QD) during the growth of the whole structure. The Mn doping was carried out by opening the Mn cell shutter during the QD growth. The cell temperature was set to $590{ }^{\circ} \mathrm{C}$. The precise determination of the $\mathrm{Mn}$ atom density is difficult in this material because of the large segregation of $\mathrm{Mn}$ atoms at these growth temperatures as observed by STM [J.-C. Girard, unpublished]. Estimations from Hall effect measurements in thick and uniformly Mn doped GaAs layers grown at the same temperature yielded a density of approximatively $1-2 \times 10^{11} \mathrm{Mn}$ atoms per $\mathrm{cm}^{2}$, giving a probability of $1 / 3-2 / 3 \mathrm{Mn}$ per dot. However, in $\mu$-PL measurements on a large collection of single QDs we observed only rare occurrences of $\mathrm{Mn}$ doping $(<0.1 \%)$, likely due to the $\mathrm{Mn}$ segregation away from the QD layer. Samples grown at higher $\mathrm{Mn}$ cell temperature $\left(660{ }^{\circ} \mathrm{C}\right)$ showed a much larger probability $(\sim 1 \%)$ of finding dots containing a single Mn atom.

The $\mu$-PL spectroscopy of individual InAs:Mn QDs was carried out with a split-coil magneto-optic cryostat. A $2 \mathrm{~mm}$ focal length aspheric lens (N.A. 0.5) was used to focus the He-Ne excitation beam and to collect the PL from the sample, while the relative positioning in all three directions was ensured by Attocube ${ }^{T M}$ piezo-motors. All measurements presented in this Letter were performed at low temperature $(\mathrm{T}=2 \mathrm{~K})$ and the magnetic field was applied parallel to the optical axis 
(Faraday configuration). The PL was dispersed by a $0.6 \mathrm{~m}$-focal length double spectrometer and detected by a Nitrogen-cooled CCD array camera.

We first present the optical signature in zero magnetic field of a single Mn atom uncovered by our experiments in the PL spectrum of about 10 different QDs. A characteristic spectrum is shown in Fig. 1(a). It consists of two bright doublets labelled $F$ and $A F$ separated by an energy $\Delta$ of the order of a few $100 \mu \mathrm{eV}$, and of a weaker central line denoted by $O$. The splitting $\delta$ of the doublets is the same for both lines $F$ and $A F$ and typically amounts to a few tens of $\mu \mathrm{eV}$. A simple
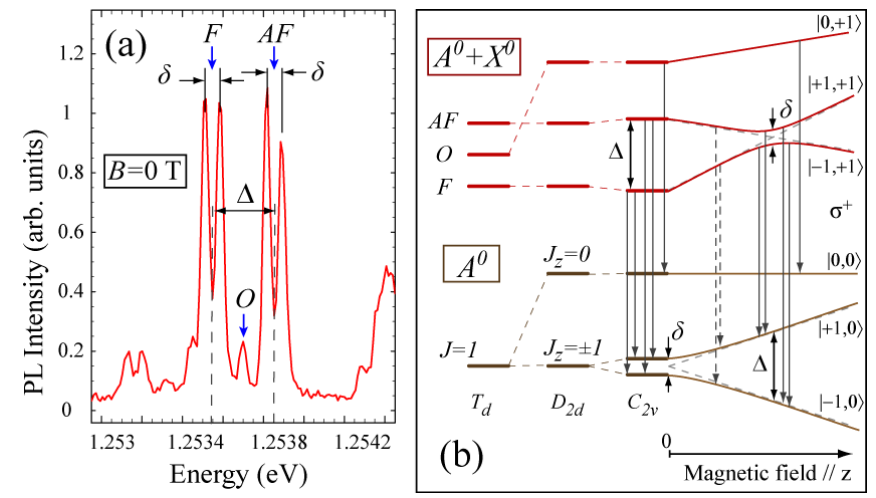

FIG. 1: (Color Online) (a) Micro-PL spectrum of an individual InAs quantum doped with a single $\mathrm{Mn}$ atom in zero magnetic field at $T=2 \mathrm{~K}$. These lines were identified as originating from a charged exciton $X^{-}$. (b) Schematic of the excitonic transitions from $A^{0}+X^{0}$ (initial state) to $A^{0}$ (final state) taking into account the local potential anisotropy and a longitudinal magnetic field. For simplicity only the bright states corresponding to $\sigma^{+}$polarized transitions are shown. The same diagram holds for $A^{0}+X^{-}$(see text) by replacing the above labels by $\left|J_{z},+3 / 2\right\rangle\left(\left|J_{z},+1 / 2\right\rangle\right)$ in the initial (final) states respectively.

interpretation of this spectral feature can be constructed by assuming that the $\mathrm{QD}$ contains a Mn impurity in the $A^{0}$ configuration, i.e. a hole bound to an $A^{-}$ center [16, 17, 18]. This acceptor state is characterized in bulk GaAs by an anti-ferromagnetic " $p$ - $d$ " exchange between the $3 d^{5} \mathrm{Mn}$ spin $S=5 / 2$ and the hole spin $J_{h}=3 / 2$. The latter takes the form of a Heisenberg Hamiltonian $\varepsilon \hat{\boldsymbol{S}} \cdot \hat{\boldsymbol{J}}_{\boldsymbol{h}}$ [17 (with $\varepsilon \sim 5 \mathrm{meV}$ ), giving rise to splittings of $A^{0}$ eigenstates as a function of the total angular momentum $\hat{\boldsymbol{J}}=\hat{\boldsymbol{J}}_{\boldsymbol{h}}+\hat{\boldsymbol{S}}$ : the triplet ground state $J=1\left(J_{z}= \pm 1,0\right)$ turns out to be well separated from the higher levels $J=2,3,4$ by at least $2 \varepsilon \sim 10 \mathrm{meV}$. Therefore, at low temperature when $k_{B} T \ll 2 \varepsilon$ the Mn impurity is completely thermalized in its ground state. Assuming similar " $p$ - $d$ " exchange in InAs QDs, we may consider that the photo-carriers captured by the QD interact with an effective spin $J=1$. Such a situation is depicted in Fig. 1(b) in the case of an electron-hole pair (or neutral exciton $X^{0}$ ) in its ground state. We focus here only on the exciton bright states with projection of angular momentum $J_{z}^{e x c}$. $= \pm 1$, since the dark states $\left(J_{z}^{e x c}= \pm 2\right)$ do not contribute to the PL signal. A natural basis to describe the $A^{0}+X^{0}$ initial states of the excitonic transition reads thus $\left|J_{z}, J_{z}^{\text {exc. }}\right\rangle$. In case of exchange interaction between $A^{0}$ and $X^{0}$, these levels are split into three doubly degenerate levels which read $| \pm 1, \pm 1\rangle,|0, \pm 1\rangle,|\mp 1, \pm 1\rangle$ corresponding to a ferromagnetic $(F)$, "orthogonal" $(O)$, and anti-ferromagnetic $(A F)$ spin configuration, respectively. If the final $A^{0}$ states were perfectly degenerate, as predicted for the acceptor level in bulk GaAs, then we should observe in the PL spectrum three lines equally spaced and of identical intensity, similar to the six lines observed in CdTe QDs doped with a single Mn atom [12. Actually, due to its hole component, the $A^{0}$ state is sensitive to local variations of composition and strain over a typical distance of $1 \mathrm{~nm}$ from the impurity center [16, 18, 19]. In self-assembled InAs QDs which ressemble a flat lens of $\sim 4 \mathrm{~nm}$ height, the most important perturbation of the bulk potential occurs along the growth direction, $z$. Such a perturbation of $D_{2 d}$ symmetry shifts the $J_{z}=0$ level to higher energy with respect to the $J_{z}= \pm 1$ states. The same effect occurs for the $O$ level in the $A^{0}+X^{0}$ complex as this perturbation is diagonal in the basis we have chosen. If in addition the potential experienced by the impurity has some in-plane anisotropy (with $C_{2 v}$ symmetry or lower), then the $J_{z}= \pm 1$ are further split by an energy $\delta$. Such an effect is expected because the Mn impurity is very likely not in the center of the QD. Note that this anisotropy acts perturbatively as an off-diagonal term for the $A^{0}+X^{0}$ levels which are already split by $\Delta$. Following this scheme, the optical transitions from the $F$ and $A F$ levels appear as doublets due to the final state splitting, whereas the $O$ level may recombine only to the $J_{z}=0$ state because of orthogonality of its $A^{0}$ component $\left(J_{z}=0\right)$ with the $J_{z}= \pm 1$ states. The shift of the $O$ level does not reflect in the transition energy, since it appears in both the initial and final states, however it explains the weaker intensity observed experimentally for the $O$ line because of $A^{0}$ thermalization in the $J_{z}= \pm 1$ levels.

The main support to this interpretation comes from the evolution in a longitudinal magnetic field. Thanks to the Zeeman effect it is possible to restore the $A^{0}$ eigenstates to $J_{z}= \pm 1$. The $F$ and $A F$ doublets should transform to single lines for a field $B_{z}>\delta / 2 g_{1} \mu_{\mathrm{B}}$ where $g_{1}$ is the $A^{0} g$ factor in the $J=1$ spin configuration and $\mu_{\mathrm{B}}$ is the Bohr magneton. Taking the value $g_{1}=2.77$ found for GaAs:Mn [16] the typical magnetic field required amounts to only $230 \mathrm{mT}$ for the $\mathrm{QD}$ shown in Fig. 1. In parallel, the magnetic field splits the $F$ and $A F$ levels by the sum of Zeeman effects for $A^{0}$ and $X^{0}$. Therefore, the Zeeman splitting of $A^{0}$ does not reflect straightforwardly in the PL spectra apart for the "forbidden" transitions involving a spin-flip of $A^{0}$ and represented by dashed arrows in Fig. 1(b). When the 
magnetic field reaches the value $\Delta / 2 g_{1} \mu_{\mathrm{B}}(\sim 1 \mathrm{~T}$ in our case) the $|+1,+1\rangle$ and $|-1,+1\rangle$ states are now brought into coincidence. Since they are formed with the same exciton spin, the anisotropic interaction between the $J_{z}= \pm 1$ levels splits the $A^{0}+X^{0}$ levels by the same energy splitting $\delta$ as in zero field. For this very specific field the PL spectrum should thus be quite similar to the spectrum in zero field as illustrated in Fig. 1 (b), with the splitting $\Delta(\delta)$ in the final (initial) states.

To study the magnetic field dependence of the
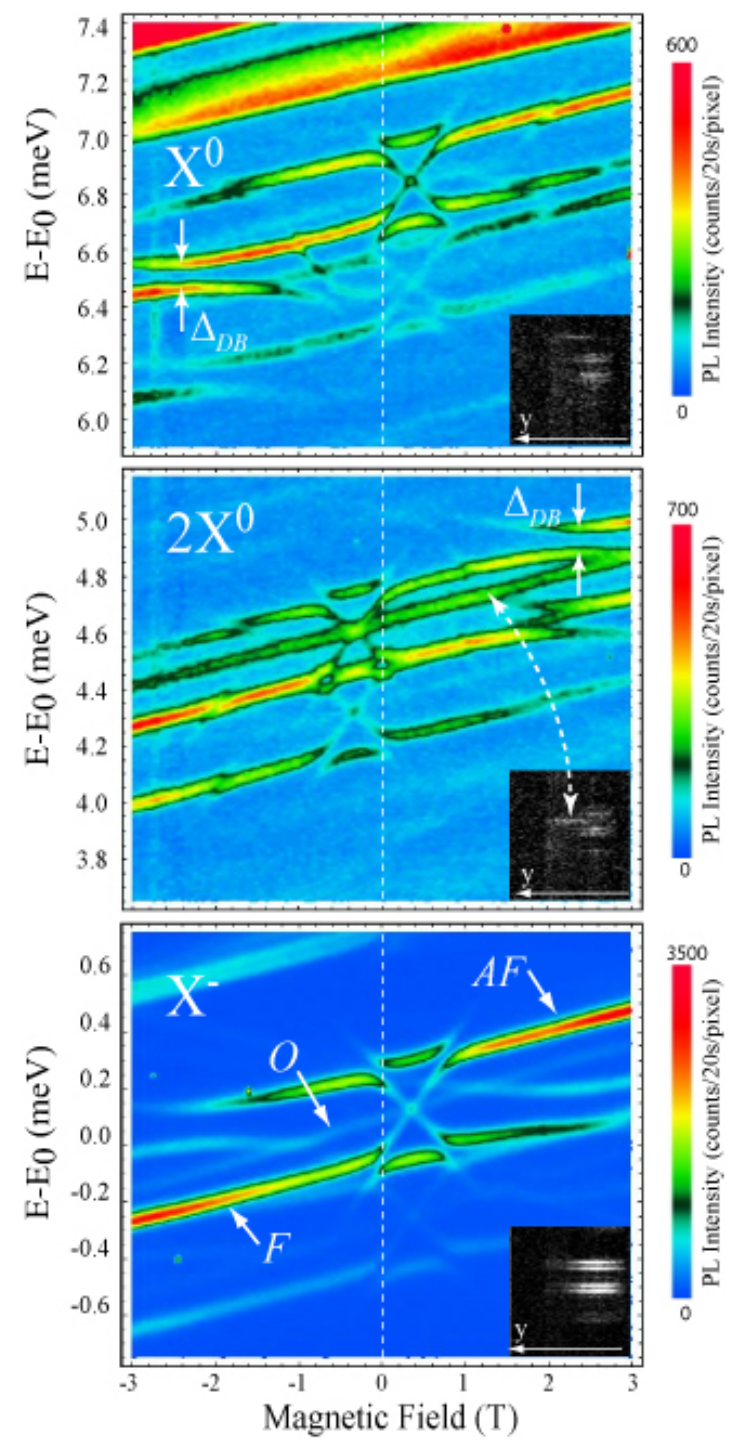

FIG. 2: (Color online) Contour-plot of $\mu$-PL intensity from a single InAs QD doped with a single Mn impurity against longitudinal magnetic field and detection energy $E-E_{0}\left(E_{0}=\right.$ $1.2536 \mathrm{eV})$. The three energy windows correspond to the $X^{0}$, $2 X^{0}$ and $X^{-}$states observed in the same $\mu$-PL spectra. Insets : CCD images (100 pixels $\times \sim 150$ pixels) at $B_{z}=0 \mathrm{~T}$ showing the spatial correlations of the lines along the vertical axis $y$ of the set-up. An extra line coming from another QD (dashed arrow) is superposed on the $2 X^{0}$ feature.

$X^{0}$-to- $A^{0}$ coupling, we recorded a series of $121 \mu$-PL spectra over a 10 meV-energy range, by varying the magnetic field from $-3 \mathrm{~T}$ to $+3 \mathrm{~T}$ with a step of $50 \mathrm{mT}$. The detection was set $\sigma^{+}$to help identify the different levels and their interactions. The $\mu$-PL intensity was plotted on a color-scale against magnetic field and energy-detection, using an interpolating function for graphical rendering. To focus on the spin-dependent interactions we subtracted the diamagnetic-shift $\propto B_{z}^{2}$. Figure 2 displays three spectral regions of this contourplot, showing clearly correlated spectral lines that could be identified (after a careful analysis) as the three excitonic features $X^{0}, 2 X^{0}$ and $X^{-}$originating all from the same individual QD. Remarkably, the $2 X^{0}$ and $X^{-}$ set of lines are separated from $X^{0}$ by roughly the same binding energies than in undoped InAs QDs emitting at $\sim 1.25 \mathrm{eV}$ [20. Note Fig. 1(a) is the cross-section at $B=0 \mathrm{~T}$ of the $X^{-}$contour-plot.

The main feature common to the plots of Fig. 2 is

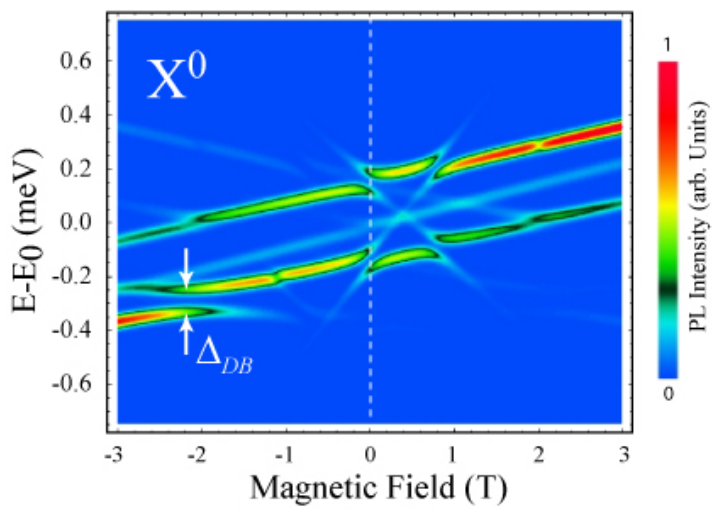

FIG. 3: (Color online) Theoretical contour-plot of $X^{0} \mathrm{PL}$ intensity from a single InAs QD doped with a single Mn impurity against longitudinal magnetic field and energy.

a very peculiar pattern resulting from the evolution of the zero-field doublets to another pair of doublets at $B \approx 0.75 \mathrm{~T}$. The resulting crossing lines correspond to the "forbidden" transitions involving $A^{0}$ spin-flip from $J_{z}= \pm 1$ to $J_{z}=\mp 1$ respectively. Obviously these transitions are not strictly forbidden because of the anisotropic coupling either in the final state (at $B=0$ ), or in the initial state (at $B \approx 0.75 \mathrm{~T}$ ). Focusing on the $X^{-}$feature, we clearly observe a strong evolution of the intensity ratio between the $F$ and $A F$ lines due to the $A^{0}$ thermalization on one of the $J_{z}= \pm 1$ levels depending on the field direction [23]. For $B_{z}>0$, the $|+1,+1\rangle(|-1,+1\rangle)$ population should decrease (increase). Actually, it is this simple feature which allowed us to ascribe confidently the low energy doublet $F$ to the ferromagnetic $A^{0}+X^{0}$ configuration. We note that such an effective ferromagnetic coupling was already reported by J. Szczytko et al. 21] in very dilute $\mathrm{Ga}_{1-\mathrm{x}} \mathrm{Mn}_{\mathrm{x}} \mathrm{As}(x<0.001)$.

In each case shown in Fig. 2, an exact replica of 
the main pattern is found at lower energy. We ascribe them to temporal electrostatic fluctuations of the QD environment which rigidly shift all the excitonic lines, e.g. due to charge trapping and detrapping in the QD vicinity. Since these replica were not found for other Mn-doped QDs that we have examined (and showing also the same cross-like patterns) we conclude that they are not related to the intrinsic signature of a Mn-impurity. We chose to show this particular dot because three excitonic complexes were simultaneously visible with a high signal to noise ratio.

Another striking feature is the symmetry between $X^{0}$ and $2 X^{0}$. It results from the polarization correlation in the biexciton cascade imposed by the Pauli principle. As we detect only $\sigma^{+}$photons the measured transitions from $2 X^{0}$ lead to the $\sigma^{-}$polarized $X^{0}$, which obviously has the same field dependence as the $\sigma^{+}$polarized $X^{0}$ but for $B_{z} \rightarrow-B_{z}$. This observation strongly supports the line identification and actually indicates that the biexciton (with both holes and electrons in singlet spin configuration) has no spin interaction with the $\mathrm{Mn}$ impurity. Note that the very same symmetry has been observed in Mn-doped CdTe QDs [13].

Finally, the position of the cross-like pattern for the $X^{-}$case is very instructive. It reveals that one of the electron- $A^{0}$ or hole- $A^{0}$ exchange integrals must be vanishingly small with respect to the other. If not the mixing at $B_{z}=0$ between the $J_{z}= \pm 1$ states would be reduced both in the initial state (due to hole- $A^{0}$ exchange) and final state (due to electron- $A^{0}$ exchange). There would be no splitting and the cross-like pattern would be shifted to a different field. Since it appears at the same positive field as for $X^{0}$, the $X^{-}$transitions must be described by the diagram of Fig. 1(b), yet with $e-A^{0}$ as the final state. We can therefore conclude that the electron- $A^{0}$ coupling is negligible as compared to $\delta$ (actually below $20 \mu \mathrm{eV}$ from a precise comparison of the spectra at $\left.B_{z}=0\right)$.

To support the above discussion, we have modeled the spin interactions with the Mn impurity for the three excitonic configurations. To reproduce all details of our experimental results, it appeared necessary to include not only the $J=1$ states of $A^{0}$ but also the $J=2$ states. Our model includes the Zeeman Hamiltonian for a single particle (Mn, bound hole $h_{1}$, QD $S$-shell hole $h_{2}$ and electron $e$ ), strain Hamiltonian for $h_{1}$ [19, valence band mixing between light- and heavy-components for $h_{2}$ [22], and exchange interaction within each pair of particles. A detailed discussion of this model will be published elsewhere. We present in Fig. 3 the contour plot of theoretical PL spectra corresponding to the $X^{0}-A^{0}$ configuration. By adjusting strain and exchange parameters, our model reproduces remarkably well the cross-like pattern, the effect of $\mathrm{Mn}$ thermalization $\left(T_{\mathrm{Mn}}=10 \mathrm{~K}\right)$ as well as the anticrossing $\Delta_{D B}$ observed at $-2 \mathrm{~T}$. The latter results from a coupling between the bright $F$ exciton $|+1,+1\rangle$ and the dark $A F$ exciton $|+1,-2\rangle$ when they are brought into coincidence by the field. Our model reveals that this is a resonant third order coupling involving the $h_{2}$ valence band mixing, a shear strain $\epsilon_{x z}$ (which also contributes to $\delta$ ) and the effective $h_{2}-A^{0}$ exchange constant $\Delta_{12}$ between the $A^{0}$ spin subspaces $J=1$ and $J=2$. It reads $\Delta_{12}=\varepsilon_{h_{2}-\mathrm{Mn}}-\varepsilon_{h_{1}-h_{2}}$ where $\varepsilon_{\alpha-\beta}$ is the exchange integral between the spins $J_{\alpha}$ and $J_{\beta}$. To reproduce our experimental results we found that the $F-A F$ splitting $\Delta$ is dominated by this exchange term $\Delta_{12}$ while the exchange term $\Delta_{11}=\left(7 \varepsilon_{h_{2}-\mathrm{Mn}}-3 \varepsilon_{h_{1}-h_{2}}\right) / 4$ within the $J=1$ subspace contributes less than $10 \%$ of $\Delta$.

In conclusion, the successful $\mu$-PL investigation in a longitudinal magnetic field of a single Mn-doped InAs quantum dots reveals remarkable features bringing new insights into the spin interactions between carriers and a Mn impurity in a III-V matrix. The anti-ferromagnetic coupling between the hole bound to the magnetic impurity and the $3 d^{5} \mathrm{Mn}$ electrons is confirmed. In contrast, the effective coupling of the Mn impurity as a whole $\left(A^{-}+h\right)$ with a hole confined in an InAs QD is proven to be ferromagnetic, while it essentially vanishes for a confined electron. The influence of the strain field on the $\mathrm{Mn}$ acceptor level is clearly evidenced, and gives rise to a very specific spectral signature of the Mn doping. Our results reveal that the $\mathrm{Mn}$ spin in $A^{0}$ configuration represents a two-level system well separated from higher energy levels which opens new outlooks for spin-based quantum information processing, e.g. by exploiting the exchange interaction with optically polarized carriers.

This work was partly supported by the European Network of Excellence SANDIE, the ANR contracts BOITQUANT and MOMES.

* Electronic address: Aristide.Lemaitre@lpn.cnrs.fr,

$\dagger$ Electronic address: Olivier.Krebs@lpn.cnrs.fr

[1] A. L. Efros et al. , Phys. Rev. Lett. 87, 206601 (2001).

[2] J. Fernandez-Rossier and R. Aguado, Phys. Rev. Lett. 98, 106805 (2007).

[3] M. N. Leuenberger and D. Loss, Nature 410, 789 (2001).

[4] M. Xiao et al. , Nature 430, 435 (2004).

[5] J. M. Elzerman et al. , Nature 430, 431 (2004).

[6] Y. Manassen et al. , Phys. Rev. Lett. 62, 2531 (1989).

[7] A. J. Heinrich et al. , Science 306, 466 (2004).

[8] C. F. Hirjibehedin et al., Science 312, 1021 (2006).

[9] D. Kitchen et al. , Nature 442, 439 (2006).

[10] D. Rugar et al. , Nature 430, 329 (2004).

[11] A. Gruber et al. , Science 276, 2012 (1997).

[12] L. Besombes et al. , Phys. Rev. Lett. 93, 207403 (2004).

[13] L. Besombes et al. , Phys. Rev. B 71, 161307 (2005).

[14] Y. Léger et al. , Phys. Rev. Lett. 95, 047403 (2005).

[15] Y. Léger et al. , Phys. Rev. Lett. 97, 107401 (2006). 
[16] J. Schneider et al. , Phys. Rev. Lett. 59, 240 (1987).

[17] A. K. Bhattacharjee and C. B. à la Guillaume, Solid State Comm. 113, 17 (1999).

[18] A. O. Govorov, Phys. Rev. B 70, 035321 (pages 5) (2004).

[19] A. M. Yakunin et al. , Nat. Mat. 6, 512 (2007).

[20] B. Eble et al., Phys. Rev. B 74, 081306(R) (2006).

[21] J. Szczytko, W. Mac, A. Stachow, A. Twardowski, P. Be- cla, and J. Tworzydlo, Solid State Comm. 99, 927 (1996).

[22] K. Kowalik et al. , Phys. Rev. B 75, 195340 (2007).

[23] Thermalization effects are assumed negligible in the excitonic states due to a long spin lifetime compared to the recombination time. See also Ref. 12 . 